\title{
Theoretical Analysis of Friction Compensation Using Sliding Mode Control
}

\author{
Rafan, N.A ${ }^{1, a}$, Jamaludin, $Z^{1, b}$, Tjahjowidodo, $T^{2, c}$, Chey, L.S., \\ and Chiew, T.H $\mathrm{H}^{1, d}$ \\ ${ }^{1}$ Department of Robotics and Automation, Faculty of Manufacturing Engineering, Universiti \\ Teknikal Malaysia Melaka, Hang Tuah Jaya,76100 Durian Tunggal, Melaka, Malaysia \\ ${ }^{2}$ School of Mechanical and Aerospace Engineering, Division Mechatronics and Design, Nanyang \\ Technological University, 50 Nanyang Avenue, North Spine (N3) Level 2, Singapore 639798, \\ Singapore \\ aaidawaty@utem.edu.my, bamberi@utem.edu.my, 'ttegoeh@ntu.edu.sg, \\ dfosterchiew87@hotmail.com
}

Keywords: Friction Compensation, Sliding Mode Control, Generalized Maxwell Slip Model

\begin{abstract}
Friction is an undesired nonlinear phenomenon that reduces position and tracking accuracy in machine tools application. This paper focuses on development of control technique to compensate friction force at motion reversal of a drive system that generates quadrant glitch phenomenon thus improving tracking accuracy. Sliding Mode Control (SMC) is designed to compensate friction. The Generalized Maxwell-Slip (GMS) friction model is applied for numerical analysis. The performance of the controller is analysed based on the reduction in the quadrant glitches magnitude. The performance of the SMC controller is compared with the classical PID controller. Results show that SMC controller yields the smallest quadrant glitch magnitudes.
\end{abstract}

\section{Introduction}

In high precision positioning applications, the effects of friction in the system can lead to significant positioning error. Spike at quadrant position observed during circular motion or most commonly known as quadrant glitches. These quadrant glitches are the product of complex nonlinear behavior at motion reversal of a motion system that can critically affects the tracking performance [1]. In order to compensate the error due to frictional forces, an effective control strategy is a necessary. This requires a deep understanding and knowledge regarding friction behavior and friction characteristic of the system. This study focuses on the characterization and modeling of friction behavior in such axis ball screw driven system. A Generalized Maxwell-Slip model (GMS) is incorporated within the system and a sliding mode control (SMC). SMC is designed to study its robustness against the non-linear effect of friction forces.

\section{Friction Characterisation and Modeling}

A GMS friction model is selected for analysis purpose due to its ability to compensate friction forces efficiently based on a physically motivated friction model [2]. Parameter identification of the GMS friction model is based on actual friction behavior of a system. Based on these identified parameters, the GMS friction model is embedded into system to simulate the friction behavior. A GMS friction model with four elementary slip-blocks is selected [1], yielding a total of 13 model parameters. The identification of the parameters of the state equation is considered at constant velocity, where motor force equals the friction force and is represents by the force control command signal. In pre-sliding regime, friction is dominated by displacement and behaves as a hysteretic function of displacement with non-local memory behavior known as virgin curve. The virgin curve is derived from a sinusoidal excitation of the system [1]. The frequency and amplitude of the sinusoidal are selected to minimize inertia effect and to remain in the pre-sliding regime. Four $\alpha_{i}^{\prime} s$ 
and $k_{i}$ 's slip-block parameters are obtained by analyzing virgin curve by using linear function. The knots and slopes determine the $\alpha_{i}^{\prime} s$ and $k_{i}$ 's parameters [6]. Table 1 summarizes the identified GMS model parameters. Other GMS model parameters $\left(F_{c}, F_{s}, V_{s}, \sigma\right.$, and $\left.\delta\right)$ are obtained from the static friction model identification that includes Coulomb friction, $F_{c}$, Stribeck force, $F_{s}$, and Stribeck velocity, $V_{s}$ and constant $\delta$.

Table 1. Identified parameters of the state equation

\begin{tabular}{|l|l|l|l|l|l|}
\hline $\begin{array}{l}\text { Static } \\
\text { friction }\end{array}$ & $F_{c}=105 N$ & $F_{s}=165 N$ & $\begin{array}{l}1 / \mathrm{V}_{\mathrm{s}}=0.001 \mathrm{~s} \\
\mu m^{-1}\end{array}$ & $\sigma=0.00004 N \mathrm{~s} / \mu m$ & $\delta=1$ \\
\hline $\begin{array}{l}\text { GMS } \\
\text { friction } \\
\text { model }\end{array}$ & $k_{1}[N / \mu \mathrm{m}]$ & $k_{2}[N / \mu m]$ & $k_{3}[N / \mu m]$ & $k_{3}[N / \mu m]$ & \\
& 99.9359 & 1.3641 & 1.0807 & 0.1193 & \\
\cline { 2 - 6 } & $\alpha_{1}=0.5663$ & $\alpha_{2}=0.2265$ & $\alpha_{3}=0.1699$ & $\alpha_{4}=0.0385$ & \\
\hline
\end{tabular}

Chattering effect of the SMC controller using signum and sigmoid like function were investigated. For tracking, friction compensation performance of the SMC controller were analysed and compared to the classical PID controller in terms of the quadrant glitches magnitude. Actual positions were compared to the reference sinusoidal signal. These numerical validations were performed using MATLAB/SIMULINK.

\section{Sliding Mode Control Design}

Sliding mode control (SMC) is a form of Variable Structure Control (VSC)[4]. SMC utilizes a high frequency switching control signal to enforce the system trajectories onto a surface, the so-called sliding surface (or hyper plane), after a finite time and remain within the vicinity of the sliding surface towards the equilibrium point thereafter. Sliding mode control (SMC) is a powerful and robust nonlinear feedback control method. Two main features of SMC are the finite reaching time and the complete disturbance rejection of matched uncertainties. In SMC applications, the system states approach the switching line (or surface) and slide along this line to reach the final states. During sliding, the control behavior is independent of the system dynamics and hence is independent of the influence of the acting disturbance forces [1]. Switching function is a main element in SMC design besides control law that determines the dynamics transient response of a system. For a second order system, the sliding surface, $s$, is a function of the tracking error, $e(t)$ and its time derivative [4]. Hence, for a simple mass-damper-spring system, without the disturbance function, $d(s)$, the control input becomes:

$$
\begin{aligned}
& u_{\text {equivalent }}=m(\ddot{r}-\lambda \dot{e})+c \dot{y}+k y \\
& u(t)=u_{\text {equivalent }}-K . \operatorname{sign}(s)
\end{aligned}
$$

Equivalent control maintains the sliding motion. The discontinuous signum function with proportional gain $K$ are the main element to keep attached to sliding surface in finite time. This discontinuous signum function, though leads to high frequency switching [4]. The states are oscillating about the surface instead of remaining on them which is normally known as chattering[5]. A continuous approximation of the discontinuous signum function is a simple alternative. A sigmoid-like function shown in 1.3 replaces the discontinuous signum function in 1.2.

$$
V_{\delta}(s)=\frac{s}{|s|+\delta}
$$

The positive constant $\delta$ illustrates the degree of the continuous approximation. Instead of having an ideal sliding motion, the states lie within a specified boundary of the sliding surface resulting in non-ideal sliding motion, also known as pseudo-sliding [4]. 


\section{Results and Discussion}

Analysis of chattering effect of SMC. The control command signals of the SMC model with signum and sigmoid-like function are analyzed and compared. Fig. 1 shows command input control signal for (a) Ideal sliding and (b) Pseudo sliding. The chattering phenomenon in ideal sliding approach is much more obvious (indicates by the red dotted circle) compared with the pseudo sliding approach.

Analysis of friction compensation. The performance of the controllers in compensating nonlinear frictional forces is validated based on reduction in magnitude of the quadrant glitches [6]. The performance of the SMC controller is compared to the classical controller. Figure 2 shows quadrant glitches with PID controller.

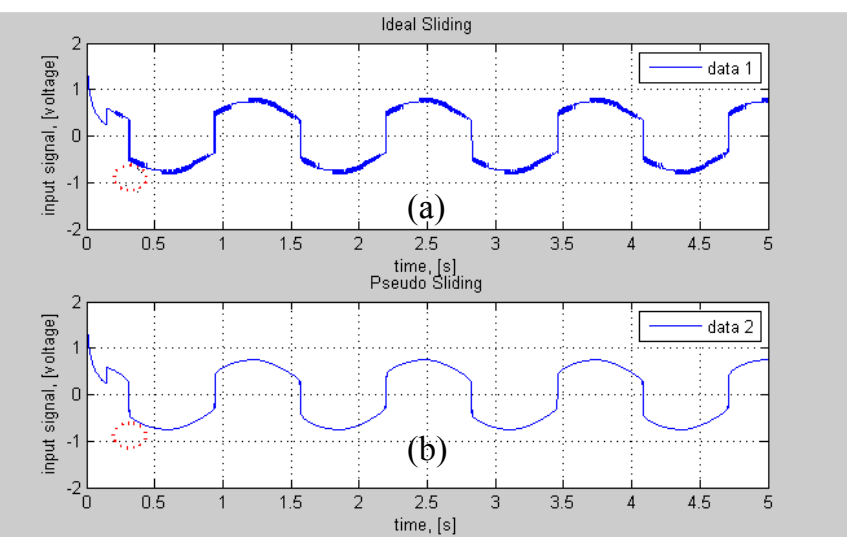

Fig. 1. Chattering phenomenon in (a) ideal sliding and (b)pseudo sliding

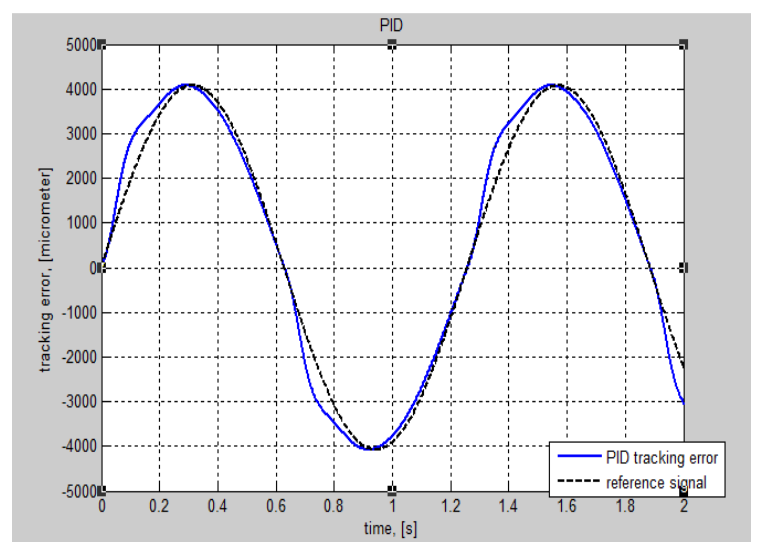

Fig. 2. Glitches from PID

The PID controller exhibits significant quadrant glitches near motion reversal compared to SMC. The magnitude of the spike formed in PID controller is about $815 \mu \mathrm{m}$. Fig. 3 presents tracking errors using ideal sliding and pseudo sliding. The magnitude of the glitch formed in the ideal sliding controller is estimated at $20 \mu \mathrm{m}$. The pseudo sliding approach reduces further the quadrant glitches magnitude to $18 \mu \mathrm{m}$. These figures also highlight chattering effect as seen from the recorded noisy characteristic of the signal.

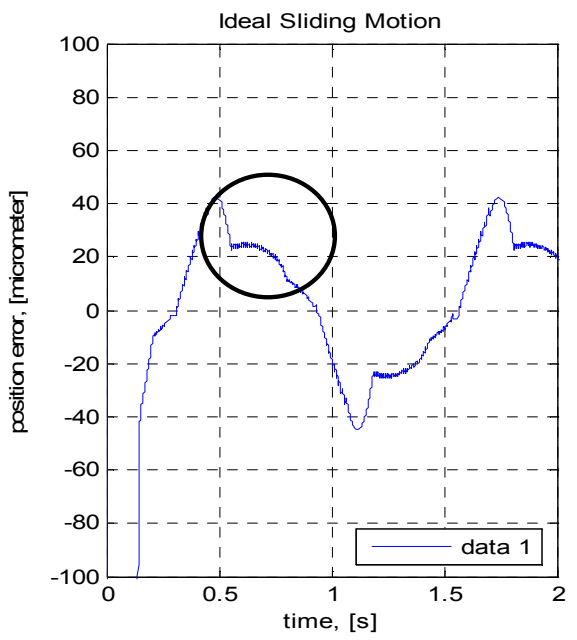

(a)

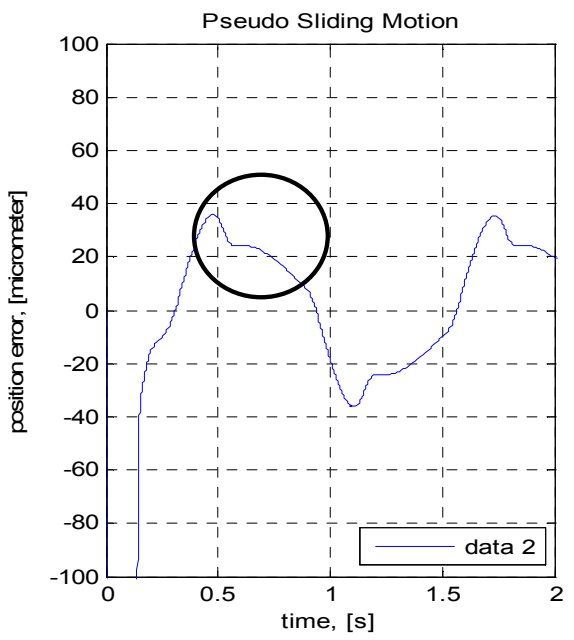

(b)

Fig. 3. Position error using (a) ideal sliding and (b) pseudo sliding

\section{Conclusion}

Numerical analyses were performed for friction compensation performance in a single axis linear drive system using PID controller and a SMC controller. A GMS friction model is incorporated into the system model to simulated actual friction behavior. This friction model was identified based on 
the actual system. SMC controller yields reduction in the magnitude of the quadrant glitches from a maximum of $815 \mu \mathrm{m}$ to $18 \mu \mathrm{m}$. This study has demonstrated the effectiveness of the SMC controller to compensate nonlinear friction behavior especially at motion reversal. The accuracy of friction model in describing the pre-sliding behavior of the friction regime determines the degree of performance in compensating this disturbance force. Further study on higher degree SMC is desirable to improve further the tracking performance.

\section{References}

[1] Jamaludin, Z., Brussel, H. V., Pipeleers, G. and Swevers, J. (2008). “Accurate Motion Control of XY High-Speed Linear Drives using Friction Model Feedforward and Cutting Forces Estimation." CIRP Annals - Manufacturing Technology. vol. 57, issue 1, pp. 403 - 406.

[2] Lampaert, V.; Al-Bender, F.; and Swevers, J. (2003). "A Generalized Maxwell-Slip Friction Model appropriate for Control Purposes." IEEE International Conference on Physics and Control Proceedings-PhysCon, St. Petersburg, Russia. 24-31.

[3] Eun-Chan, P.; Hyuk, L.; and Chong-Ho, C. (2003). "Position Control of X-Y Table at Velocity Reversal Using Presliding Friction Characteristics." IEEE Transactions on Control Systems Technology, vol. 11, pp.

[4] Jamaludin, Z.; Van Brussel, H.; and Swevers, J. (2007). "Classical cascade and sliding mode control tracking performances for a xy feed table of a high-speed machine tool." International Journal on Precision Technology, vol. 1, no. 1, pp. 65-74.

[5] Young, K.D, Utkin, V.I and Ozguner, U; (1999). "A control engineer's guide to sliding mode control”, IEEE Transactions on Control System Technology, Vol 7, No. 3.

[6] Jamaludin, Z.; Van Brussel, H.; and Swevers, J.(2008). "Quadrant Glitch Compensation using Friction Model-Based Feedforward and an Inverse-Model-Based Disturbance Observer." IEEE International Workshop On Advanced Motion Control, Torento, Italy.

[7] Ho,H.F.; Wong, Y.K.; and Rad, A.B. (2009) "Adaptive fuzzy sliding mode control with chattering elimination for nonlinear SISO systems", Simulation Modelling, Practice and Theory, vol. 17, Issue 7, pp 1199-1210.

[8] Kempf, C. J.; and Kobayashi, S. (1999) "Disturbance observer and feedforward design for a high-speed direct drive positioning table", IEEE Transactions On Control System Tech, vol. 7, no. 5, pp 513-526.

[9] Olsson, H.; Astrom, K. J.; Canudas De Witt, C.; Gafvert, M.; and Lischinsky, P. (1997). "Friction Models and Friction Compensation.", Control, vol 4, Issue 3, pp. 1-37.

[10] Sung-Won, P.; Jeong, J.; Hyun-Seok, Y.; Young-Pil, P.; and No-Cheol, P. (2005). "Repetitive Controller Design for Minimum Track Misregistration in Hard Disk Drives." IEEE Transactions on Magnetics, vol. 41, no. 9, pp. 2522-2528.

[11] Swevers, J,; Al Bender, F,; Ganseman, C.; and Prajogo, T. (2000). "An integrated friction model structure with improved pre-sliding behaviour for accurate friction compensation," IEEE Transactions on Automatic Control, vol. 45, no. 4, pp. 675-686.

[12] Tjahjowidodo, T.; Al-Bender, F.; Van Brussel, H.; and Symens, W. (2007). "Friction characterization and compensation in electro-mechanical systems." Journal of Sound and Vibration, vol 308, Issue 3-5, pp. 632-646.

[13] Chen, M. ; and Chen, W.H.; (2009). "Sliding mode control for a class of uncertain nonlinear system based on disturbance observer", International Journal of Adaptive Control and Signal Processing, vol 24, Issue 1, pp. 51-64 